\title{
A stabilizing model predictive controller for uncertain max-plus-linear systems and uncertain switching max-plus-linear systems*
}

T. van den Boom and B. De Schutter

If you want to cite this report, please use the following reference instead:

T. van den Boom and B. De Schutter, "A stabilizing model predictive controller for uncertain max-plus-linear systems and uncertain switching max-plus-linear systems," Proceedings of the 18th IFAC World Congress, Milan, Italy, pp. 8663-8668, Aug.Sept. 2011.

Delft Center for Systems and Control

Delft University of Technology

Mekelweg 2, 2628 CD Delft

The Netherlands

phone: +31-15-278.24.73 (secretary)

URL: https: //www.dcsc.tudelft.nl

*This report can also be downloaded viahttps://pub. deschutter.info/abs/11_010.html 


\title{
A stabilizing model predictive controller for uncertain max-plus-linear systems and uncertain switching max-plus-linear systems *
}

\author{
Ton van den Boom* Bart De Schutter* \\ * Delft Center for Systems and Control, Delft University of \\ Technology, Mekelweg 2, 2628 CD Delft, The Netherlands \\ a.j.j.vandenboom@tudelft.nl, b.deschutter@tudelft.nl, \\ http://dcsc.tudelft.nl
}

\begin{abstract}
We first discuss conditions for stability for uncertain max-plus-linear systems and for uncertain switching max-plus-linear systems, where in the uncertainty description the system matrices live in a union of polyhedra. Based on the newly derived stability conditions, a stabilizing model predictive controller is derived for both uncertain max-plus-linear systems and uncertain switching max-plus-linear systems.
\end{abstract}

Keywords: Discrete event and hybrid systems, max-plus-linear systems, model uncertainty, model predictive control, robust control

\section{INTRODUCTION}

The class of discrete event systems (DES) essentially consists of man-made systems that contain a finite number of resources that are shared by several users all of which contribute to the achievement of some common goal (Baccelli et al., 1992). In general, models that describe the behavior of a DES are nonlinear in conventional algebra. However, there is a class of DES that can be described by a model that is linear in the max-plus algebra. This class of max-plus-linear (MPL) systems can only characterize synchronization and no concurrency or choice. In switching MPL systems the system can switch between different modes of operation, in which the mode switching depends on a switching mechanism. In this paper we will consider the control of both uncertain MPL systems and uncertain switching MPL systems.

In contrast to conventional linear systems, where noise and disturbances are usually modeled by including an extra term in the system equations (i.e., the noise is considered to be additive), the influence of noise and disturbances in (switching) MPL systems is not max-plus-additive, but max-plus-multiplicative. This means that the system matrices will be perturbed and as a consequence the system properties will change. Ignoring the noise can lead to a bad tracking behavior or even to an unstable closed loop. A second important feature is modeling errors. Uncertainty in the modeling or identification phase leads to errors in the system matrices. It is clear that both modeling errors, and noise and disturbances perturb the system by introducing uncertainty in the system matrices. Sometimes

\footnotetext{
* Research partially funded by the Dutch Technology Foundation STW project "Model-predictive railway traffic management" (11025), and by the European 7th Framework Network of Excellence project "Highly-complex and networked systems (HYCON2)".
}

it is difficult to distinguish the two from one another, but usually fast changes in the system matrices will be considered as noise and disturbances, whereas slow changes or permanent errors are considered as model mismatch. In this paper we extend the results of van den Boom and De Schutter (2002b) and consider MPL systems and switching MPL systems in a single framework. We distinguish between two characterizations: static uncertainty, in which the system is uncertain but the system matrices are constant, and dynamic uncertainty, in which the uncertainty in the system is affected by bounded noise. We will also show that for both characterizations we can derive stability conditions under quite general assumptions.

Model predictive control (MPC) (Camacho and Bordons, 1995: Maciejowski, 2002) is a model-based predictive control approach that has its origins in the process industry and that has mainly been developed for linear or nonlinear time-driven systems. Its main ingredients are: a prediction model, a performance criterion to be optimized over a given horizon, constraints on inputs and outputs, and a receding horizon approach. We show that for MPC of (switching) MPL systems with the given uncertainty description we can use the results of van den Boom and De Schutter (2002b).

The uncertainty description in this paper is related to the interval uncertainty given by Lhommeau et al. (2004); Corronc et al. (2010). For other classes of DES uncertainty results can be found in Cardoso et al. (1999); Liu (1993); Park and Lim (1999); Young and Garg (1995), and the references therein.

This paper is organized as follows. In Section 2 we give a concise introduction to the max-plus algebra and (S)MPL systems with uncertainty. In Section 3 we derive stability conditions for (S)MPL systems with uncertainty, and in 
Section 4 we show that the MPC method derived in van den Boom and De Schutter (2002b) can be used to control uncertain (S)MPL systems.

\section{MAX-PLUS ALGEBRA AND MPL SYSTEMS}

\subsection{Max-plus algebra}

In this section we give the basic definition of the max-plus algebra (Baccelli et al., 1992; Cuninghame-Green, 1979). Define $\varepsilon=-\infty$ and $\mathbb{R}_{\varepsilon}=\mathbb{R} \cup\{\varepsilon\}$. The max-plus-algebraic addition $(\oplus)$ and multiplication $(\otimes)$ are defined as follows:

$$
x \oplus y=\max (x, y) x \otimes y=x+y
$$

for any $x, y \in \mathbb{R}_{\varepsilon}$, and

$$
\begin{aligned}
& {[A \oplus B]_{i j}=A_{i j} \oplus B_{i j}=\max \left(A_{i j}, B_{i j}\right)} \\
& {[A \otimes C]_{i j}=\bigoplus_{k=1}^{n} A_{i k} \otimes C_{k j}=\max _{k=1, \ldots, n}\left(A_{i k}+C_{k j}\right)}
\end{aligned}
$$

for matrices $A, B \in \mathbb{R}_{\varepsilon}^{m \times n}$ and $C \in \mathbb{R}_{\varepsilon}^{n \times p}$. The matrix $\varepsilon$ is the max-plus-algebraic zero matrix: $[\varepsilon]_{i j}=\varepsilon$ for all $i, j$.

A max-plus diagonal matrix $S=\operatorname{diag}_{\oplus}\left(s_{1}, \ldots, s_{n}\right)$ has elements $S_{i j}=\varepsilon$ for $i \neq j$ and diagonal elements $S_{i i}=s_{i}$ for $i=1, \ldots, n$. The max-plus identity matrix is equal to $E=\operatorname{diag}_{\oplus}(0, \ldots, 0)$. The max-plus-algebraic matrix power of a matrix $A \in \mathbb{R}_{\varepsilon}^{n \times n}$ is defined as follows: $A^{\otimes^{0}}=E$ and $A^{\otimes^{k}}=A \otimes A^{\otimes^{k-1}}$ for $k=1,2, \ldots$. If for a max-plus diagonal matrix $S=\operatorname{diag}_{\oplus}\left(s_{1}, \ldots, s_{n}\right)$ all values $s_{i}$ are finite we find that the inverse of $S$ is equal to $S^{\otimes^{-1}}=$ $\operatorname{diag}_{\oplus}\left(-s_{1}, \ldots,-s_{n}\right)$. There holds $S \otimes S^{\otimes}=S^{\otimes^{-1}} \otimes$ $S=E$.

\section{Definition 1. Lunze (1997)}

The structure matrix $P^{\mathrm{s}}$ of a matrix $P \in \mathbb{R}_{\varepsilon}^{n \times m}$ is an element of $\{0, \varepsilon\}^{n \times m}$, that is defined as follows: for all $i=1, \ldots, n$, and $j=1, \ldots, m$, if $P_{i j}=\varepsilon$ then $P_{i j}^{\mathrm{s}}=\varepsilon$ and if $P_{i j} \neq \varepsilon$ then $P_{i j}^{\mathrm{s}}=0$.

Obviously, a structure matrix describes a class of matrices with the same $\varepsilon$-structure but different finite elements.

Let $\mathcal{S}_{\mathrm{mps}}$ be the set of functions $f$ defined as:

$$
f(x)=\max _{i}\left(\alpha_{i, 1} x_{1}+\ldots+\alpha_{i, n} x_{n}+\beta_{i}\right)
$$

with $x \in \mathbb{R}_{\varepsilon}^{n}$ and $\alpha_{i, j} \in \mathbb{R}^{+}$and $\beta_{i} \in \mathbb{R}$. We write $f \in \mathcal{S}_{\text {mps }}(x)$, if we want to stress that $f$ is a function of $x$.

Lemma 2. (van den Boom and De Schutter, 2002b) The set $\mathcal{S}_{\mathrm{mps}}$ is closed under the operations $\oplus, \otimes$, and scalar multiplication by a nonnegative scalar.

Let $A=\left[\begin{array}{llll}a_{1} & a_{2} & \cdots & a_{n}\end{array}\right]$, then

$$
\operatorname{vec}(A)=\left[\begin{array}{c}
a_{1} \\
a_{2} \\
\vdots \\
a_{n}
\end{array}\right]
$$

Finally, let $\left\{H_{1}, H_{2}, \ldots, H_{n}\right\}$ be matrices with an identical $\varepsilon$-structure. Then the convex closure $\mathrm{Co}\left(H_{1}, \ldots, H_{n}\right)$ is the smallest convex set containing $H_{1}, \ldots, H_{n}$. So $\mathrm{Co}\left(H_{1}, \ldots, H_{n}\right)=\left\{H \mid H=\lambda_{1} H_{1}+\lambda_{2} H_{2}+\ldots+\right.$ $\left.\lambda_{n} H_{n}, \quad 0 \leq \lambda_{m} \leq 1, \sum_{m} \lambda_{m}=1\right\}$, where for $\varepsilon$ entries we use the convention:

$$
\lambda_{1} \varepsilon+\lambda_{2} \varepsilon+\ldots+\lambda_{n} \varepsilon=\varepsilon, \text { for } \lambda_{m} \geq 0
$$

\subsection{MPL systems and uncertainty}

In Cuninghame-Green (1979); Baccelli et al. (1992) it has been shown that DES in which there is synchronization but no choice can be described by a model of the form

$$
\begin{aligned}
& x(k)=A(k) \otimes x(k-1) \oplus B(k) \otimes u(k) \\
& y(k)=C(k) \otimes x(k) .
\end{aligned}
$$

Systems that can be described by this model will be called max-plus-linear (MPL) systems. The index $k$ is called the event counter. For DES the state $x(k)$ typically contains the time instants at which the internal events occur for the $k$ th time, the input $u(k)$ contains the time instants at which the input events occur for the $k$ th time, and the output $y(k)$ contains the time instants at which the output events occur for the $k$ th time.

Assumption A1: The uncertainty is modeled as follows. Define the matrix

$$
H(k)=\left[A(k) B(k) C^{T}(k)\right] \quad \in \mathbb{R}_{\varepsilon}^{n \times\left(n+n_{u}+n_{y}\right)}
$$

and let

$$
H(k) \in \mathcal{H}=\mathcal{H}^{(1)} \cup \mathcal{H}^{(2)} \cup \ldots \cup \mathcal{H}^{(L)}
$$

where $\mathcal{H}^{(\ell)}, \ell=1, \ldots, L$ are sets of uncertain system matrices with each set having a specific $\varepsilon$-structure. The sets $\mathcal{H}^{(\ell)}, \ell=1, \ldots, L$ are assumed to be polyhedra, defined by

$$
\mathcal{H}^{(\ell)}=\operatorname{Co}\left(H^{(\ell, 1)}, H^{(\ell, 2)}, \ldots, H^{\left(\ell, n_{\ell}\right)}\right)
$$

where

$H^{(\ell, m)}=\left[A^{(\ell, m)} B^{(\ell, m)}\left(C^{(\ell, m)}\right)^{T}\right] \in \mathbb{R}_{\varepsilon}^{n \times\left(n+n_{u}+n_{y}\right)}$ for $\ell=1, \ldots, L, m=1, \ldots, n_{\ell}$. Note that all matrices $H=\left[\begin{array}{lll}A & B & C^{T}\end{array}\right]$ in the set $\mathcal{H}^{(\ell)}$ have the same structure matrix $H_{\ell}^{\mathrm{s}}=\left[\begin{array}{lll}A_{\ell}^{\mathrm{s}} & B_{\ell}^{\mathrm{s}} & \left(C^{\mathrm{s}}\right)_{\ell}^{T}\end{array}\right]$ for all $\ell=1, \ldots, L$.

The choice of this uncertainty model is motivated as follows:

- For ordinary MPL systems with $L=1$ all the uncertain system matrices have the same $\varepsilon$-structure and the uncertainty is mainly because of variation in the finite parameters, such as varying processing times, transportation times, etc. This uncertainty description was already discussed in van den Boom and De Schutter (2002b). For $L>1$ the $\varepsilon$-structure of the uncertain system may change as well.

- We can model uncertainty of switching MPL systems in which the system properties as event order and synchronization between events may change.

For each $k$ we can find parameters $\mu_{\ell m}(k)$ where $0 \leq$ $\mu_{\ell m}(k) \leq 1$ such that

$$
H(k)=\sum_{\ell=1}^{L} \sum_{m=1}^{n_{\ell}} \mu_{\ell m}(k) H^{(\ell, m)}
$$


If $H(k) \in \mathcal{H}^{(i)}$ then

$$
\sum_{m=1}^{n_{\ell}} \mu_{\ell m}(k)= \begin{cases}1 & \text { for } \ell=i \\ 0 & \text { for } \ell \neq i\end{cases}
$$

If $H(k)=H \in \mathcal{H}$ is constant for all $k$ we talk about static uncertainty. This static uncertainty then only contains static model errors.

If $H(k) \in \mathcal{H}$ may vary for every event $k$ we talk about dynamic uncertainty. The uncertainty may contain some noise as well as (dynamic and/or static) model errors.

\section{CONDITIONS FOR STABILITY}

Just like in van den Boom and De Schutter (2002a), we adopt the notion of stability for DES from Passino and Burgess (1998), in which a DES is called stable if all its buffer levels remain bounded. All the buffer levels in DES are bounded if the dwelling times of the parts or batches in the system remain bounded. This implies for an uncertain (switching) MPL system with a due date signal $r$ that closed-loop stability is achieved if there exist finite constants $k_{0}, M_{y r}, M_{y x}$ and $M_{x u}$ such that

$$
\begin{aligned}
\left|y_{i}(k)-r_{i}(k)\right| & \leq M_{y r}, & \forall i \\
\left|y_{i}(k)-x_{j}(k)\right| & \leq M_{y x}, & \forall i, j \\
\left|x_{j}(k)-u_{m}(k)\right| & \leq M_{x u}, & \forall j, m
\end{aligned}
$$

for all $k>k_{0}$. Condition (3) means that the delay between the actual output date $y(k)$ and the due date $r(k)$ remains bounded, and on the other hand, that the stock time will remain bounded. Conditions (4) and (5) mean that the throughput time (i.e. the time between the starting date $u(k)$ and the output date $y(k))$ is bounded. For a due date defined as

$$
r(k)=\rho k+d(k), \text { where }\left|d_{i}(k)\right| \leq d_{\max }, \forall i, k
$$

where $r$ and $d$ are vectors signals and $\rho$ is a scalar, satisfying $\rho>0$, this implies finite buffer levels.

Similar to deterministic (switching) MPL systems, stability is not an intrinsic feature of the uncertain (switching) MPL system, but it also depends on the due dates (i.e., the reference signal) of the system. In van den Boom and De Schutter (2002a) we already observed that for MPL systems, the largest max-plus-algebraic eigenvalue of the system matrix $A$ gives an upper bound on the asymptotic slope of the due date sequence. For uncertain (switching) MPL systems we cannot directly use the largest max-plusalgebraic eigenvalue, but we use the concept of maximum growth rate:

Definition 3. Consider an uncertain (switching) MPL system satisfying Assumption A1. Define for a given scalar $\alpha$ the matrices $A_{\alpha}^{(\ell, m)}$ as $\left[A_{\alpha}^{(\ell, m)}\right]_{i j}=\left[A^{(\ell, m)}\right]_{i j}-\alpha$. The maximum growth rate $\lambda$ of the uncertain (switching) MPL system is the smallest $\alpha$ for which there exists a max-plus diagonal matrix $S=\operatorname{diag}_{\oplus}\left(s_{1}, \ldots, s_{n}\right)$ with finite diagonal elements $s_{i}$, such that

$$
\left[S \otimes A_{\alpha}^{(\ell, m)} \otimes S^{-1}\right]_{i j} \leq 0, \quad \forall i, j, \ell, m
$$

Remark 1: Note that for any uncertain (switching) MPL system the maximum growth rate $\lambda$ is finite, or more precisely:

$$
\lambda \leq \max _{i, j, \ell, m}\left[A^{(\ell, m)}\right]_{i j}
$$

This fact is easily verified by noting that for $\lambda^{\prime}=$ $\max _{i, j, \ell, m}\left[A^{(\ell, m)}\right]_{i j}$, and using the max-plus identity matrix $S=\operatorname{diag}_{\oplus}(0, \ldots, 0)$ one has

$$
\left[S \otimes A_{\lambda^{\prime}}^{(\ell, m)} \otimes S^{\otimes^{-1}}\right]_{i j}=\left[A_{\lambda^{\prime}}^{(\ell, m)}\right]_{i j}=\left[A^{(\ell, m)}\right]_{i j}-\lambda^{\prime} \leq 0
$$

for all $i, j, \ell, m$. In general the maximum growth rate $\lambda$ can be easily computed by solving a linear programming problem (van den Boom and De Schutter, 2007).

Lemma 4. For an uncertain (switching) max-plus-linear system with

$$
A(k)=\sum_{\ell=1}^{L} \sum_{m=1}^{n_{\ell}} \mu_{\ell m} A^{(\ell, m)}
$$

and a matrix $S$ satisfying equation (7) we find that

$$
\left[S \otimes A_{\alpha}(k) \otimes S^{\otimes}\right]_{i j} \leq 0, \quad \forall i, j
$$

This property immediately follows from the fact that $A_{\alpha}(k)$ is a convex combination of $A_{\alpha}^{(\ell, m)}$.

Let the set $\mathcal{L}_{N}=\left\{\left[\ell_{1} \cdots \ell_{N}\right]^{T} \mid \ell_{m} \in\{1, \ldots, L\}, m=\right.$ $1, \ldots, N\}$ represent the set of all possible permutations of $N$ numbers from the set $\{1, \ldots, L\}$.

Definition 5. Let the matrix $\left[A_{\ell}^{\mathrm{s}} B_{\ell}^{\mathrm{s}}\left(C_{\ell}^{\mathrm{s}}\right)^{T}\right]$ be the structure matrix for the set $\mathcal{H}^{(\ell)}$ for all $\ell \in\{1, \ldots, L\}$. An uncertain (switching) MPL system is weakly controllable if there exists a finite positive integer $N$ such that for all $\ell \in\{1, \ldots, L\}$ the matrices

$$
\Gamma^{N}(\ell)=\left[\begin{array}{lll}
B_{\ell}^{\mathrm{s}} & A_{\ell}^{\mathrm{s}} \otimes B_{\ell}^{\mathrm{s}} \ldots\left(A^{\mathrm{s}}\right)_{\ell}^{\otimes(N-1)} \otimes B_{\ell}^{\mathrm{s}}
\end{array}\right]
$$

are row-finite, i.e. in each row there is at least one entry larger then $\varepsilon$.

An uncertain (switching) MPL system is strongly controllable if there exists a finite positive integer $N$ such that for all $\tilde{\ell}=\left[\ell_{1} \cdots \ell_{N}\right]^{T} \in \mathcal{L}_{N}$ the matrices

$$
\begin{array}{r}
\Gamma^{N}(\tilde{\ell})=\left[\begin{array}{lll}
B_{\ell_{N}}^{\mathrm{s}} & A_{\ell_{N}}^{\mathrm{s}} \otimes B_{\ell_{N-1}}^{\mathrm{s}} & A_{\ell_{N}}^{\mathrm{s}} \otimes A_{\ell_{N-1}}^{\mathrm{s}} \otimes B_{\ell_{N-2}}^{\mathrm{s}} \\
\cdots & A_{\ell_{N}}^{\mathrm{s}} \otimes A_{\ell_{N-1}}^{\mathrm{s}} \otimes \ldots \otimes A_{\ell_{2}}^{\mathrm{s}} \otimes B_{\ell_{1}}^{\mathrm{s}}
\end{array}\right]
\end{array}
$$

are row-finite, i.e. in each row there is at least one entry larger then $\varepsilon$.

In the following theorem we prove that if the system is controllable and the slope of the due-date signal $r$ is not too steep for the system to follow, and we allow the input to only vary in a bounded way around the due date signal, the system is stable.

Theorem 6. Consider the uncertain (switching) MPL system (1)-(2) satisfying Assumption A1, with a maximum growth rate $\lambda$ and due-date signal (6). Define the matrices $A_{\rho}^{(\ell, m)}$ with $\left[A_{\rho}^{(\ell, m)}\right]_{i j}=\left[A^{(\ell, m)}\right]_{i j}-\rho$. Further assume $C^{(\ell, m)}$ to be row-finite. Let $v_{\max }$ be any finite positive number. Now if

(1) $\rho \geq \lambda$

(2) the system is weakly controllable,

then any input signal

$$
u(k)=\rho k+v(k), \text { where }\left|v_{i}(k)\right| \leq v_{\max }, \forall i, k,
$$

will stabilize the (switching) MPL system with static uncertainty. 
If the system is also strongly controllable, then any input signal

$$
u(k)=\rho k+v(k), \text { where }\left|v_{i}(k)\right| \leq v_{\max }, \forall i, k,
$$

will stabilize the (switching) MPL system with dynamic uncertainty.

Proof : First note that condition (10) holds if and only if there exists a diagonal matrix $S$ with finite entries such that

$$
\left[S \otimes A_{\rho}^{(\ell, m)} \otimes S^{-1}\right]_{i j} \leq 0, \quad \forall i, j, \ell, m .
$$

From this it follows that

$$
\left[S \otimes A_{\rho}(k) \otimes S^{\otimes}\right]_{i j} \leq 0, \forall i, j .
$$

where $A_{\rho}(k)$ is such that $\left[A_{\rho}(k)\right]_{i j}=[A(k)]_{i j}-\rho$.

Let $S$ be the diagonal matrix with finite diagonal elements, such that (10) is satisfied, and define the signals

$$
\begin{aligned}
z(k) & =S \otimes(x(k)-\rho k) \\
w(k) & =y(k)-\rho k \\
v(k) & =u(k)-\rho k
\end{aligned}
$$

and the matrices

$$
\begin{aligned}
\bar{A}_{\rho}(k) & =S \otimes A_{\rho}(k) \otimes S^{\otimes} \\
\bar{B}(k) & =S \otimes B(k) \\
\bar{C}(k) & =C(k) \otimes S^{\otimes^{-1}} .
\end{aligned}
$$

To every $\rho$ we can associate a shifted system (van den Boom et al., 2005)

$$
\begin{aligned}
z(k) & =\bar{A}_{\rho}(k) \otimes z(k-1) \oplus \bar{B}(k) \otimes v(k) \\
w(k) & =\bar{C}(k) \otimes z(k) .
\end{aligned}
$$

It is easy to verify that if (11) holds, this shifted system is also weakly controllable. Stability means that all signals in this system should remain bounded. In other words, we have to prove that there exist finite values $z_{\max }, w_{\max }$, such that

$$
|z(k)| \leq z_{\max } \quad, \quad|w(k)| \leq w_{\max }
$$

Now consider the uncertain (switching) MPL system (15)(16) satisfying Assumption A1, for the input signal $v_{i}(k) \leq$ $v_{\max }, \forall i, k$. Let $z_{\max }(k)=\max _{i} z_{i}(k)$ and ${ }^{1} \bar{b}_{\max }=$ $\max _{i, j, \ell, m}\left(\left[\bar{B}^{(\ell, m)}\right]_{i j}\right)$, then by $(15)$ we have

$$
\begin{aligned}
z_{i}(k)=\max \left(\max _{j}\left(\left[\bar{A}_{\rho}(k)\right]_{i j}+z_{j}(k-1)\right),\right. \\
\left.\max _{j}\left([\bar{B}(k)]_{i j}+v_{j}(k)\right)\right) \\
\leq \max \left(\max _{j}\left(\left[\bar{A}_{\rho}(k)\right]_{i j}\right)+z_{\max }(k-1),\right. \\
\left.\max _{i, j, \ell, m}\left(\left[\bar{B}^{(\ell, m)}\right]_{i j}\right)+v_{\max }\right) \\
\leq \max \left(z_{\max }(k-1), \bar{b}_{\max }+v_{\max }\right)
\end{aligned}
$$

where we use the fact that $\left[\bar{A}_{\rho}\right]_{i j}(k) \leq 0$ because of $(14)$. Hence

$$
z_{\max }(k) \leq \max \left(z_{\max }(k-1), \bar{b}_{\max }+v_{\max }\right)
$$

This means that all entries of the shifted state $z(k)$ have a non-increasing signal $z_{\max }(k)$ as an upper bound.

\footnotetext{
1 Note that $\bar{b}_{\max }$ is finite due to the fact that the shifted system is weakly controllable.
}

Now again consider the uncertain (switching) MPL system (15)-(16) satisfying Assumption A1, for the input signal $v_{i}(k) \geq-v_{\max }, \forall i, k$. Recall that the shifted system is controllable. This implies that there is an $N$ such that

$$
\begin{aligned}
\gamma_{i} & =\max _{j}\left(\left[\bigoplus_{t=1}^{N} \bar{A}_{\rho}(k+m-1)\right.\right. \\
& \otimes \bar{A}_{\rho}(k+m-2) \otimes \ldots \otimes \bar{A}_{\rho}(k+m-N+t+1) \\
& \left.\otimes \bar{B}(k+m-N-t)]_{i j}\right)
\end{aligned}
$$

is finite. A lower bound for $\gamma_{i}$ can be found by taking the $\alpha$ to be the smallest possible finite entry of all the matrices $\bar{A}_{\rho}(k+m)$ and $\beta$ to be the smallest possible finite entry of all possible matrices $\bar{B}(k+m)$. Then

$$
\gamma_{\min }=\min _{t=0, \ldots, N}(\beta+t \alpha)=\min (\beta, N \alpha+\beta) \leq \gamma_{i}
$$

is finite. By successive substitution we find that for any $m \geq N$ there holds

$$
\begin{aligned}
& {[z(k+m)]_{i}=\left[\bar{A}_{\rho}(k+m) \otimes z(k+m-1) \oplus\right.} \\
& \bar{B}(k+m) \otimes v(k+m)]_{i} \\
& =\left[\bar{A}_{\rho}(k+m) \otimes \bar{A}_{\rho}(k+m-1) \otimes z(k+m-2) \oplus\right. \\
& \bar{B}(k+m) \otimes v(k+m-N) \\
& \left.\bar{A}_{\rho}(k+m) \otimes \bar{B}(k+m) \otimes v(k+m-N)\right]_{i} \\
& =\left[\bar{A}_{\rho}(k+m) \otimes \bar{A}_{\rho}(k+m-1) \otimes \ldots \otimes\right. \\
& \left.\bar{A}_{\rho}(k+m-N+1) \otimes z(k+m-N)\right]_{i} \oplus \\
& {[\bar{B}(k+m) \otimes v(k+m)} \\
& \bar{A}_{\rho}(k+m) \otimes \bar{B}(k+m-1) \otimes v(k+m-1) \oplus \\
& \bar{A}_{\rho}(k+m) \otimes \bar{A}_{\rho}(k+m-1) \otimes \ldots \otimes \\
& \bar{B}(k+m-N+1) \otimes v(k+m-N+1)]_{i} \\
& =\left[\bar{A}_{\rho}(k+m) \otimes \bar{A}_{\rho}(k+m-1) \otimes \ldots\right. \\
& \left.\otimes \bar{A}_{\rho}(k+m-N+2) \otimes z(k+m-N)\right]_{i} \oplus \\
& {\left[\bigoplus_{t=1}^{N} \bar{A}_{\rho}(k+m) \otimes \bar{A}_{\rho}(k+m-1) \otimes \ldots\right.} \\
& \otimes \bar{A}_{\rho}(k+m-N+t+1) \otimes \bar{B}(k+m-N+t) \otimes \\
& \otimes v(k+m-N+t)]_{i} \\
& \geq\left[\bigoplus_{t=1}^{N} \bar{A}_{\rho}(k+m-1) \otimes \bar{A}_{\rho}(k+m-2) \otimes \ldots\right. \\
& \otimes \bar{A}_{\rho}(k+m-N+t-1) \otimes \bar{B}(k+m-N+t) \otimes \\
& \otimes v(k+m-N+t)]_{i} \\
& \geq \max _{j}\left(\left[\bigoplus_{t=1}^{N} \bar{A}_{\rho}(k+m-1) \otimes \bar{A}_{\rho}(k+m-2) \otimes \ldots\right.\right. \\
& \left.\otimes \bar{A}_{\rho}(k+m-N+t-1) \otimes \bar{B}(k+m-N+t)\right]_{i j} \\
& \left.+v_{j}(k+m-N+t)\right) \\
& \geq \gamma_{i}-v_{\max } \\
& \geq \gamma_{\min }-v_{\max }
\end{aligned}
$$

We conclude that after $N$ event steps we have a lower bound for our shifted state $z(k)$. Let $\bar{c}_{\max }$ and $\bar{c}_{\min }$ be the largest and the smallest finite values of $\bar{C}^{(\ell, m)}, \forall \ell, m$, respectively. Now from (16) it follows that

$$
\left.w_{i}(k)=\max _{j}\left(\left[\bar{C}_{\rho}(k)\right]_{i, j}+z_{j}(k)\right]\right)
$$

and so after $N$ event steps $w(k)$ will be bounded by 


$$
\begin{aligned}
\gamma_{\min }-v_{\max }+ & \bar{c}_{\min } \leq w_{i}(k) \\
& \leq \max \left(z_{\max }(k-1), \bar{b}_{\max }+v_{\max }\right)+\bar{c}_{\max }
\end{aligned}
$$

where $z_{\max }(\cdot)$ is a non-increasing signal.

Define $\zeta(k)=S \otimes(r(k)-\rho k)=S \otimes d(k)$ and $s_{\max }=$ $\max _{p}\left(s_{p}\right)$ and $s_{\text {min }}=\min _{p}\left(s_{p}\right)$. Then $\left|\zeta_{i}(k)\right| \leq \zeta_{\max }=$ $s_{\max }+d_{\max }$. For any $k>N$, there holds

$$
\begin{aligned}
& y_{i}(k)-r_{i}(k)= \\
& =\left[\left(S^{\otimes} \otimes w(k)\right)+\rho k\right]_{i}-\left[\left(S^{\otimes} \otimes \zeta(k)\right)+\rho k\right]_{i} \\
& =\left(-s_{i}+w_{i}(k)+\rho k\right)-\left(-s_{i}+\zeta_{i}(k)+\rho k\right) \\
& =w_{i}(k)-\zeta_{i}(k) \\
& \leq \max \left(z_{\max }(0), \bar{b}_{\max }+v_{\max }\right)+\bar{c}_{\max }+s_{\max }+d_{\max } \\
& =M_{y r 1} \text {, } \\
& r_{i}(k)-y_{i}(k)=\zeta_{i}(k)-w_{i}(k) \\
& \leq d_{\max }+s_{\max }-\gamma_{\min }+v_{\max }-\bar{c}_{\min } \\
& =M_{y r 2} \text {, } \\
& \left|y_{i}(k)-r_{i}(k)\right|=\left|w_{i}(k)-\zeta_{i}(k)\right| \\
& \leq \max \left(M_{y r 1}, M_{y r 2}\right)=M_{y r}<\infty, \\
& y_{i}(k)-x_{j}(k)= \\
& =\left[\left(S^{\otimes} \otimes w(k)\right)+\rho k\right]_{i}-\left[\left(S^{\otimes^{-1}} \otimes z(k)\right)+\rho k\right]_{j} \\
& =\left(-s_{i}+w_{i}(k)+\rho k\right)-\left(-s_{j}+z_{j}(k)+\rho k\right) \\
& =w_{i}(k)-z_{j}(k)+\left(s_{j}-s_{i}\right) \\
& \leq \max \left(z_{\max }(0), \bar{b}_{\max }+v_{\max }\right)+\bar{c}_{\max } \\
& -\gamma_{\min }+v_{\max }+s_{\max }-s_{\min } \\
& =M_{y x}<\infty \text {, } \\
& x_{j}(k)-u_{m}(k)= \\
& =\left[\left(S^{\otimes} \otimes z(k)\right)+\rho k\right]_{j}-\left[\left(S^{\otimes} \otimes v(k)\right)+\rho k\right]_{m} \\
& =\left(-s_{j}+z_{j}(k)+\rho k\right)-\left(-s_{m}+v_{m}(k)+\rho k\right) \\
& =z_{j}(k)-v_{m}(k)+\left(s_{m}-s_{j}\right) \\
& \leq \max \left(z_{\max }(0), \bar{b}_{\max }+v_{\max }\right)+v_{\max }+s_{\max }-s_{\min } \\
& =M_{x u}<\infty \text {, }
\end{aligned}
$$

In a similar way as for $y_{i}(k)-x_{j}(k)$ and $x_{j}(k)-u_{m}(k)$ we can prove that $x_{j}(k)-y_{i}(k)$ and $u_{m}(k)-x_{j}(k)$ are bounded. This proves stability for the uncertain (switching) MPL system (1)-(2).

\section{A STABILIZING ROBUST MODEL PREDICTIVE CONTROLLER}

In De Schutter and van den Boom (2001) we have extended the MPC approach to MPL systems minimizing the criterion

$$
J(k)=\sum_{j=0}^{N_{\mathrm{p}}-1} \max (y(k+j)-r(k+j), 0)-\lambda u(k+j)
$$

In the perturbed case we have to solve the worst-case MPC problem at event step $k$ as follows:

$$
J_{\mathrm{wc}}(k)=\max _{H(k+1), \ldots, H\left(k+N_{\mathrm{p}}\right) \in \mathcal{H}} J(k, H)
$$

subject to

$$
\begin{aligned}
& u(k+j)-u(k+j-1) \geq 0 \text { for } j=0, \ldots, N_{\mathrm{p}}-1 \\
& |u(k+j)-\rho(k-j)| \leq v_{\max } \text { for } j=0, \ldots, N_{\mathrm{p}}-1 \\
& F(k) \tilde{u}(k) \leq g(k),
\end{aligned}
$$

where (17) denotes the worst-case criterion with $J(k, H)$ as short notation for $J\left(k, H(k+1), \ldots, H\left(k+N_{\mathrm{p}}\right)\right)$, (18) guar- antees a non-decreasing input signal $u$, (19) guarantees stability, and (20) denotes additional linear constraints (De Schutter and van den Boom, 2001; van den Boom and De Schutter, 2002b) with

$$
\tilde{u}(k)=\left[\begin{array}{llll}
u^{T}(k) & u^{T}(k+1) & \cdots & u^{T}\left(k+N_{\mathrm{p}}-1\right)
\end{array}\right]^{T} .
$$

Define the vector $e(k)=\operatorname{vec}(H(k))$ and let $H(k) \in \mathcal{H}^{(\ell(k)}$, then

$$
\begin{gathered}
e(k) \in E^{(\ell(k))}=\operatorname{Co}\left(\operatorname{vec}\left(H^{(\ell(k), 1)}\right), \operatorname{vec}\left(H^{(\ell(k), 2)}\right),\right. \\
\left.\ldots, \operatorname{vec}\left(H^{\left(\ell(k), n_{\ell(k)}\right)}\right)\right)
\end{gathered}
$$

Now denote the finite entries of $e(k)$ and $E^{(\ell(k))}$ by $e_{\mathrm{f}}(k)$ and $E_{\mathrm{f}}^{(\ell(k))}$, respectively. If we stack all $e_{\mathrm{f}}(k)$ we obtain

$$
\tilde{e}(k)=\left[\begin{array}{c}
e_{\mathrm{f}}(k) \\
e_{\mathrm{f}}(k+1) \\
\vdots \\
e_{\mathrm{f}}\left(k+N_{\mathrm{p}}-1\right)
\end{array}\right] \in \tilde{E}^{(\tilde{\ell}(k))}=\left[\begin{array}{c}
E_{\mathrm{f}}^{(\ell(k))} \\
E_{\mathrm{f}}^{(\ell(k+1))} \\
\vdots \\
E_{\mathrm{f}}^{\left(\ell\left(k+N_{\mathrm{p}}-1\right)\right)}
\end{array}\right]
$$

Let $\tilde{E}_{\mathrm{v}}^{(\tilde{\ell}(k))}$ contain the vertices of the polytope $\tilde{E}^{(\tilde{\ell}(k))}$.

With this notation the worst-case criterion becomes

$$
J_{\mathrm{wc}}(k)=\max _{\tilde{\ell}(k) \in \mathcal{L}^{N \mathrm{p}}} \max _{\tilde{e} \in \tilde{E}(\tilde{\ell}(k))} J(k, H(\tilde{e}))
$$

where $H(\tilde{e})$ denotes that $H(k+1), \ldots, H\left(k+N_{\mathrm{p}}\right)$ are parametrized by the entries of $\tilde{e}(k)$.

In van den Boom and De Schutter (2002b) we have proven that the criterion $J(k)$ is a max-plus-positivescaling function (see Section 1 ) in the variables $\tilde{u}$ and $\tilde{e}$ and that for a given $\tilde{\ell}(k)$ the function

$$
J_{\mathrm{wc}}(k)=\max _{\tilde{e} \in \tilde{E}(\tilde{\ell}(k))} J(k, H(\tilde{e}))
$$

can be computed by evaluating the function in the vertices of $\tilde{E}^{(\tilde{\ell}(k))}$, so

$$
J_{\mathrm{wc}}(k)=\max _{\tilde{e} \in \tilde{E}_{\mathrm{v}}^{(\tilde{\ell}(k))}} J(k, H(\tilde{e}))
$$

Furthermore this function is convex in $\tilde{u}$. This means that the optimization

$$
\min _{\tilde{u}(k)} J_{\mathrm{wc}}(k)=\min _{\tilde{u}(k)} \max _{\tilde{\ell}(k) \in \mathcal{L}^{N_{\mathrm{p}}}} \max _{\tilde{e} \in \tilde{E}_{\mathrm{v}}^{(\tilde{\ell}(k))}} J(k, H(\tilde{e}))
$$

is convex in $\tilde{u}$.

Theorem 7. Consider the uncertain (switching) MPL system (1)-(2) satisfying Assumption A1, with a maximum growth rate $\lambda$ and due-date signal (6) with $\rho \geq \lambda$. Assume the system to be controllable (weakly controllable in the case of static uncertainty, strongly controllable in the case of dynamic uncertainty) and let $C^{(\ell, m)}$ be row-finite $\forall \ell, m$. Let $v_{\max }$ be a positive constant. Now the worstcase (switching) MPL-MPC problem for event step $k$ can be defined as:

$$
\min _{\tilde{u}(k)} J_{\mathrm{wc}}(k)
$$

subject to (18)-(20). The resulting controller will stabilize the system.

The proof follows immediately from the results in this Section and previous Section.

Proposition 8 .

Let $\tilde{\ell}=\left\{\ell_{1}, \ell_{2}, \ldots, \ell_{m}\right\}$ and $\tilde{\imath}=\left\{i_{1}, i_{2}, \ldots, i_{m}\right\}$. The 
worst-case (switching) MPL-MPC problem of Theorem 7 can be recast into the following linear programming problem:

$$
\min _{\tilde{u}(k), t(k)} \max _{\tilde{\ell}, \tilde{\imath}} \sum_{j=1}^{N_{\mathrm{p}}} t_{j, \tilde{\ell}, \tilde{\imath}}(k)-\beta \tilde{u}_{j}(k)
$$

subject to

$$
\begin{aligned}
& t_{j, \tilde{\ell}, \tilde{\imath}} \geq[\tilde{C}(\tilde{\ell}, \tilde{\imath})]_{(j+1) l}+x_{l}(k-1)-r(k+j), \quad \forall j, l, \tilde{\ell}, \tilde{\imath} \\
& t_{j, \tilde{\ell}, \tilde{\imath}} \geq[\tilde{D}(\tilde{\ell}, \tilde{\imath})]_{(j+1) l}+\tilde{u}_{l}(k)-r(k+j), \quad \forall j, l, \tilde{\ell}, \tilde{\imath} \\
& t_{j, \tilde{\ell}, \tilde{\imath}} \geq 0, \quad \forall j, m \\
& u(k+j)-u(k+j-1) \geq 0, \quad \forall j \\
& -\mu_{\max } \leq u(k+j)-\rho k \leq \mu_{\max }, \quad \forall j \\
& F(k) \tilde{u}(k) \leq g(k)
\end{aligned}
$$

where $\tilde{C}$ and $\tilde{D}$ are defined as follows:

$$
\begin{aligned}
& {[\tilde{C}(\tilde{\ell}, \tilde{\imath})]_{m l}=} {\left[C^{(\ell(m), i(m))} \otimes A^{(\ell(m), i(m))}\right.} \\
&\left.\otimes \ldots \otimes A^{(\ell(1), i(1))}\right]_{l} \\
& {[\tilde{D}(\tilde{\ell}, \tilde{\imath})]_{m l}= \begin{cases}C^{(\ell(m), i(m))} \otimes A^{(\ell(m), i(m))} & \text { if } m>l \\
A^{(\ell(l), i(l+1))} \otimes B^{(\ell(l), i(l))} & \text { if } m=l \\
C^{(\ell(m), i(m))} \otimes B^{(\ell(m), i(m))} & \text { if } m<l \\
\varepsilon & \end{cases} }
\end{aligned}
$$

Proof : If we define

with

$$
t_{j, \tilde{\ell}, \tilde{\imath}}=\max (y(k+j)-r(k+j), 0)
$$

$$
y(k+j)=[\tilde{C}(\tilde{\ell}, \tilde{\imath})]_{j+1} \otimes x(k-1) \oplus[\tilde{D}(\tilde{\ell}, \tilde{\imath})]_{j+1} \otimes \tilde{u}(k)
$$

where $[\tilde{C}(\tilde{\ell}, \tilde{\imath})]_{m}$ and $[\tilde{D}(\tilde{\ell}, \tilde{\imath})]_{m}$ stand for the $m$ th row of $[\tilde{C}(\tilde{\ell}, \tilde{\imath})]$ and $[\tilde{D}(\tilde{\ell}, \tilde{\imath})]$, respectively, then (30) with $(23)$ implements (22), and (24),(25),(26) implement (31).

\section{DISCUSSION}

In this paper we have considered the control of uncertain max-plus-linear systems and uncertain switching maxplus-linear systems. The uncertainty is given in the form of the union of polytopic sets for the system matrices. These multiple sets can be used if the $\varepsilon$-structure of the system is uncertain or may change during operation. The description can also be used to describe uncertainty in switching maxplus linear systems. If the system is controllable and the slope of the due-date signal is larger or equal to than the maximum growth rate, the system will be stable if the input signal is in a bounded set. We have derived a stabilizing model predictive controller for both maxplus-linear systems as well as for switching max-plus-linear systems. For both cases the resulting optimization problem can be solved using linear programming algorithms.

In future research we would like to include constraints on the outputs, and extend the results to a probabilistic uncertainty framework. Further we will characterize the computational complexity of the worst-case MPC prob- lem, and look for ways to further improve the efficiency by complexity reduction or by approximation.

\section{REFERENCES}

Baccelli, F., Cohen, G., Olsder, G., and Quadrat, J. (1992). Synchronization and Linearity. John Wiley \& Sons, New York.

Camacho, E. and Bordons, C. (1995). Model Predictive Control in the Process Industry, Advances in Industrial Control. Springer, London.

Cardoso, J., Valette, R., and Dubois, D. (1999). Possibilistic Petri nets. IEEE Transactions on Systems, Man and Cybernetics, Part B: Cybernetics, 29(5), 573-582.

Corronc, E.L., Cottenceau, B., and Hardouin, L. (2010). Control of uncertain (max,+)-linear systems in order to decrease uncertainty. In Workshop on Discrete Event Systems, 410-415. Berlin, Germany.

Cuninghame-Green, R. (1979). Minimax Algebra, volume 166 of Lecture Notes in Economics and Mathematical Systems. Springer-Verlag, Berlin.

De Schutter, B. and van den Boom, T. (2001). Model predictive control for max-plus-linear discrete event systems. Automatica, 37(7), 1049-1056.

Lhommeau, M., Hardouin, L., Cottenceau, B., and Jaulin, L. (2004). Interval analysis and dioid: application to robust controller design for timed event graphs. Automatica, 40(11), 1923-1930.

Liu, F. (1993). Robust and adaptive supervisory control of discrete event systems. IEEE Transactions on Automatic Control, 38(12), 1848-1852.

Lunze, J. (1997). Regelungstechnik. SpringerVerlag, Berlin.

Maciejowski, J. (2002). Predictive Control with Constraints. Prentice Hall, Pearson Education Limited, Harlow, UK.

Park, S. and Lim, J. (1999). Fault-tolerant robust supervisor for discrete event systems with model uncertainty and its application to a workcell. IEEE Transactions on Robotics and Automation, 15(2), 386-391.

Passino, K. and Burgess, K. (1998). Stability Analysis of Discrete Event Systems. John Wiley \& Sons, Inc., New York, USA.

van den Boom, T. and De Schutter, B. (2002a). Properties of MPC for max-plus-linear systems. European Journal of Control, 8(5), 53-62.

van den Boom, T. and De Schutter, B. (2007). Stabilizing controllers for randomly switching max-plus-linear discrete event systems. In Proceedings of the European Control Conference 2007, 4952-4959. Kos, Greece.

van den Boom, T., De Schutter, B., and Necoara, I. (2005). On MPC for max-plus-linear systems: Analytic solution and stability. In Proceedings of the CDC/ECC 2005. Sevilla, Spain.

van den Boom, T.J. and De Schutter, B. (2002b). MPC for perturbed max-plus-linear systems. Systems and Control Letters, 45(1), 21-33.

Young, S. and Garg, V. (1995). Model uncertainty in discrete event systems. SIAM Journal on Control and Optimization, 33(1), 208-226. 